\title{
À propos de la politique des diagnostics - Raconter au lieu de compter'
}

\author{
Peter Müller-Locher
}

\author{
Psychotherapie-Wissenschaft 8 (1) 53-54 2018 \\ www.psychotherapie-wissenschaft.info \\ CC BY-NC-ND \\ DOI: $10.30820 / 8242.10$
}

Mots-clés : Fiabilité, concept relationnel, mathématique, littérature, politique

L'auteur démontre comment les efforts d'une fiabilité plus élevée dans les entretiens diagnostiques risquent de sacrifier leur qualité de liberté de narration au profit d'une exploration standardisée $\mathrm{du} / \mathrm{de} \mathrm{la} / \mathrm{des}$ patient(e)(s) et de suivre l'air du temps du comptage. À titre d'exemple, il se sert de l'expérience en tant que responsable de formation de la communauté de conseillers dans une étude de recherche. On y trouve au centre la procédure du Diagnostic psychodynamique opérationnalisé (OPD-2) dans lequel il a été essayé d'obtenir une forte concordance d'évaluation entre les différents diagnosticiens.

Quel est le lien du diagnostic psychique avec la politique ? Quel est le lien de la politique avec le diagnostic psychique ? Et quelles sont les différences que peuvent faire la narration et le comptage en ce qui concerne le diagnostic psychologique d'une part et concernant une politique de diagnostic psychologique d'autre part ? Et enfin, quel est le lien de la narration d'histoires en tant que littérature et le calcul avec des chiffres en tant que mathématique avec le diagnostic psychique et voire même avec la politique?

Les conceptions de l'auteur concernant ces questions de principe et à grande portée s'appuient sur des expériences concrètes et très limitées en tant que psychodiagnosticien dans le cadre de l'étude de cas Psychothérapie ambulatoire Suisse (PAP-S). Il s'agit d'un projet de recherche entre temps terminé de la Charte suisse de Psychothérapie en coopération avec la Clinique universitaire de Cologne et le département de Psychologie Appliquée de l'Institut supérieur Zürcher des Sciences Appliquées Zurich. 81 différents psychothérapeutes appartenant à neuf différentes écoles thérapeutiques ont laissé analyser pendant sept ans les processus thérapeutiques de 362 patient(e)s dans un concept commun de recherche (von Wyl, A. et coll. 2016).

Une kyrielle de données ont été collectées avec ces analyses. Elles se composent de questionnaires, remplis par les patient(e)s et les psychothérapeutes, et d'enre-

1 Le titre de la réflexion reprend une pensée de Jonas Lüscher (auteur et philosophe) qui atteste à notre pensée une tendance au comptage, tandis que lui en tant qu'écrivant privilégie la narration. En effet, la narration nie la complexité du monde moins que ne le fait chaque théorie mathématique. gistrements audio de la plupart des heures de thérapie. Tous(tes) les patient(e)s ont par ailleurs été invité(e)s par des assesseurs ou diagnosticiens dans ce qu'on appelle un Assessment-Center au début et à la fin de la thérapie ainsi qu'une année après l'achèvement.

Pendant 40 demi-journées d'ateliers, plus de vingt conseillers en psychothérapie impliqués en tant que diagnosticiens externes dans les différents Assessment-Centers ont essayé d'obtenir une bonne concordance dans leurs constatations médicales. Pour le diagnostic, divers questionnaires ainsi que les Entretiens structurés cliniques SKID-I et SKID-II et le diagnostic psychodynamique opérationnalisé $(\mathrm{OPD}-2)^{2}$ ont été utilisés. Le défi d'analyser au début, à la fin et un an après l'achèvement avec ces procédés de mesure et d'estimation les patient(e)s participants, afin d'évaluer l'effet de leur thérapie également d'un point de vue externe, fut considérable. Les appréciations devaient au final toutefois occasionner des constatations indépendantes des personnes à tous les moments de collecte, et notamment dans une perspective triple.

Cette concordance d'appréciation généralement recherchée comme critère de qualité et à atteindre n'a été malheureusement obtenue que dans une certaine mesure pour l'entretien OPD spécifiquement conçu. On a pu toutefois constater le gain, que

$>$ l'apprentissage mutuel organisé de façon autonome et conçu d'une manière intervisuelle dans les ateliers,

$>$ les réflexions plus longues, explicatives et orientées vers les ressources,

2 « Le Diagnostic psychodynamique opérationnalisé a la forme d'un système aux axes multiples. L'OPD repose sur les axes Vécu de la maladie et conditions préalables au traitement (I), Relations (II), Conflit (III), Structure (IV) ainsi que des troubles psychiques et psychosomatiques visés au chapitre $\mathrm{V}(\mathrm{F})$ de l'ICD-10. Après un premier entretien de une à deux heures, le clinicien (ou le chercheur) peut estimer la dynamique psychique du patient sur ces axes et renseigner dans le questionnaire d'évaluation (2006 quatrième de couverture OPD-2). » Le nouveau système de l'OPD a aussi été développé ces dernières années par un large groupe de psychothérapeutes travaillant avec une approche psychodynamique et issus des domaines de la psychanalyse, de la psychosomatique, de la psychiatrie et de la psychothérapie psychologique, et qui essaie de formuler l'opérationnalisation des aspects psychodynamiques en lien avec la thérapie. » $(2006$, p. 9) « 60 heures de formation (réparties en trois cours sur trois dates) sont nécessaires pour pouvoir utiliser le manuel d'une manière fiable. » $(2006$, p. 8) 
> la reconnaissance commune, digne de diversités dans l'estimation,

> la tentative de créer un tableau d'ensemble en prose sur la souffrance des interviewés et

- la réflexion ultérieure sur les résonances qui apparaissent dans le cercle de la communauté des conseillers ont généré à plusieurs reprises des liens sensoriels subjectifs et ont révélé des structures propres de signification dans la compréhension de l'estimation personnelle.

Par ailleurs, il est clair qu'un diagnostic psychologique à orientation quantitative qui ne décrit pas seulement le phénomène mental, mais qui au final porte ses fruits, décide surtout de l'accès à des organismes d'assurance et de cette façon se situe obligatoirement au service des décisions politiques de répartition.

Un diagnostic psychologique qui se veut être une nar- ration réduit en revanche moins la complexité confuse d'une vie spirituelle compréhensible, mais également inintelligible. Il conduit au contraire plutôt à une reconnaissance de ce que vivent les personnes qui souffrent.

\section{L'auteur}

Peter Müller-Locher, Dr. phil., Msc en développement organisationnel, est psychothérapeute psychologique résident (ASP), analyste de l'existence, superviseur et analyste de groupes. Il est président de la Commission de l'Assurance-Qualité de la Charte Suisse de Psychothérapie et participe à l'étude de cas Psychothérapie ambulatoire (PAP-S) dans différentes charges.

\section{Contact}

peter.mueller-locher@bluewin.ch 